\title{
Alterações no líquido cefalorraquidiano em pacientes com fibromialgia
}

\section{Changes in cerebrospinal fluid in patients with fibromyalgia}

\author{
Louana Cassiano da S. Lima', Marcelo Moraes Valença ${ }^{2}$ \\ 'Doutoranda do Programa de Pós-graduação em Neuropsiquiatria e Ciências do Comportamento, Universidade \\ Federal de Pernambuco, Recife, Brasil. Bolsista da Facepe \\ ${ }^{2}$ Coordenador do Programa de Pós-graduação em Neuropsiquiatria e Ciências do Comportamento, \\ Universidade Federal de Pernambuco, Recife, Brasil \\ Lima LC, Valença MM. Alterações no líquido cefalorraquidiano em pacientes com fibromialgia. \\ Headache Medicine. 2015;6(1)6-11
}

\begin{abstract}
RESUMO
Fibromialgia é uma doença crônica de etiologia desconhecida e tem a dor como principal característica. Níveis dos neurotransmissores e neuropeptídios encontrados no líquido cefalorraquidiano de indivíduos com fibromialgia podem informar a atividade dos neurônios do sistema nervoso central. O objetivo deste estudo foi observar, na literatura existente, as principais alterações de neurotransmissores e neuropeptídeos que ocorrem no líquido cefalorraquidiano de indivíduos com fibromialgia. Realizou-se uma busca bibliográfica nas bases de dados SCIELO, LILACS e PubMed, sem restrição de ano e nos idiomas português, inglês e espanhol por meio das palavras chave "fibromyalgia" e "cerebrospinal fluid". Foram encontrados 120 artigos e, após análise, 14 foram incluídos na pesquisa. Observou-se que muitas das substâncias estudadas apresentam relação com a fibromialgia, o que corrobora com algumas pesquisas que definem a fibromialgia como uma doença do sistema nervoso central.
\end{abstract}

Palavras-chave: Fibromialgia; Líquido cefalorraquidiano

\section{ABSTRACT}

Fibromyalgia is a chronic disorder with unknown etiology and pain is the main characteristic. Cerebrospinal fluid levels of neurotransmitters and neuropeptides can give information about the neuronal activity of central nervous system in patients with fibromyalgia. The objective of this research is to review the main alterations of neurotransmitters and neuropeptides in cerebrospinal fluid in patients with fibromyalgia. ScieLo, Lilacs and PubMed databases were searched, without year limitation, in Portuguese, English and Spanish idioms, using the keywords "fibromyalgia" and "cerebrospinal fluid". 120 studies were found and, after analysis, 14 were included in this research. Some substances were relates to fibromyalgia, which is in agreement with some researches that define fibromyalgia as a central nervous system disorder.

Keywords: Fibromyalgia; Cerebrospinal fluid

\section{INTRODUÇÃO}

A fibromialgia é uma doença crônica que está presente em parcela significante da população, afetando mais o gênero feminino, com cerca de 4-9 mulheres para cada homem. . $^{(1,2)}$ Sua etiologia ainda é desconhecida, porém parece haver influência de fatores genéticos e ambientais. ${ }^{(3)}$ A principal característica da fibromialgia é o baixo limiar para dor, ${ }^{(4)}$ que geralmente está associada à rigidez matinal, distúrbios do sono, depressão, ansiedade, síndrome das pernas inquietas, síndrome do intestino irritável, bem como problemas de memória e concentração. ${ }^{(1,3,5)}$ A presença de cefaleia em pacientes com fibromialgia é muito alta, sugerindo haver mecanismos fisiopatogênicos entre essas doenças, particularmente na migrânea crônica.

Estudos mostram que pacientes com fibromialgia apresentam alterações no sistema nervoso central (SNC). Alguns sugerem que exista um aumento da atividade, ou uma hipersensibilidade de vias nociceptivas, associada ou não à hipofunção dos mecanismos endógenos de 
analgesia. ${ }^{(6)}$ Outros estudos apontam como causa concentrações alteradas de neurotransmissores, ${ }^{(7,8)}$ neuropeptídeos, ${ }^{(9,10)}$ distúrbio no eixo hipotálamo-hipófise-

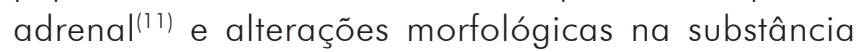
cinzenta cerebral. ${ }^{(12)}$ Tais achados sugerem que a fibromialgia deve ser interpretada como uma doença do sistema nervoso central.

Há fortes indícios que pacientes com fibromialgia apresentem um desequilíbrio entre mecanismos excitatórios e inibitórios do controle da dor, decorrente do comprometimento do sistema modulatório da dor no SNC. Esse desequilíbrio leva a uma percepção inadequada tanto de estímulos ambientais como de estímulos internos. ${ }^{(13)}$

Para que nosso sistema nervoso funcione de maneira adequada, é necessário que as concentrações de neurotransmissores e neuropeptídios estejam dentro de níveis considerados normais. Em condições que envolvem dor crônica, muitos deles encontram-se com níveis alterados, de modo a perturbar o correto funcionamento de nossas funções. Diversas substâncias que são liberadas na fenda sináptica já foram analisadas, seja por meio de análises sanguíneas, seja por meio de análises do líquido cefalorraquidiano (LCR).

O presente estudo tem por objetivo revisar as principais alterações de neurotransmissores e neuropeptídeos que ocorrem no LCR de indivíduos com fibromialgia.

\section{MÉTODO}

Realizou-se uma busca bibliográfica nas bases de dados ScieLo, Lilacs e PubMed, sem restrição de ano de publicação, utilizando-se as palavras chave "fibromyalgia" e "cerebrospinal fluid". Foram contemplados na pesquisa artigos publicados nos idiomas português, inglês e espanhol.

Para serem incluídos no presente estudo, os artigos deveriam avaliar indivíduos que preencheram os critérios diagnósticos da fibromialgia, de acordo com o Colégio Americano de Reumatologia de $1990^{(14)}$ e os critérios de 2010, ${ }^{(15)}$ a fim de mensurar os níveis de neurotransmissores e neuropeptídeos no LCR.

Os artigos foram selecionados após a leitura do título e dos resumos. Foram excluídos os artigos duplicados, as revisões de literatura, aqueles que não apresentavam grupo controle, aqueles que utilizavam modelos animais e os que analisavam outros tipos de substâncias que não neurotransmissores e neuropeptídeos.

\section{RESULTADOS}

Foi encontrado um total de 120 artigos nas bases de dados mencionadas, dos quais 60 deles eram duplicados e outros 11 não se encontravam de acordo com os idiomas escolhidos para busca. Dos 49 artigos restantes, após leitura criteriosa dos títulos e resumos, foram selecionados 14 para análise (Figura 1).

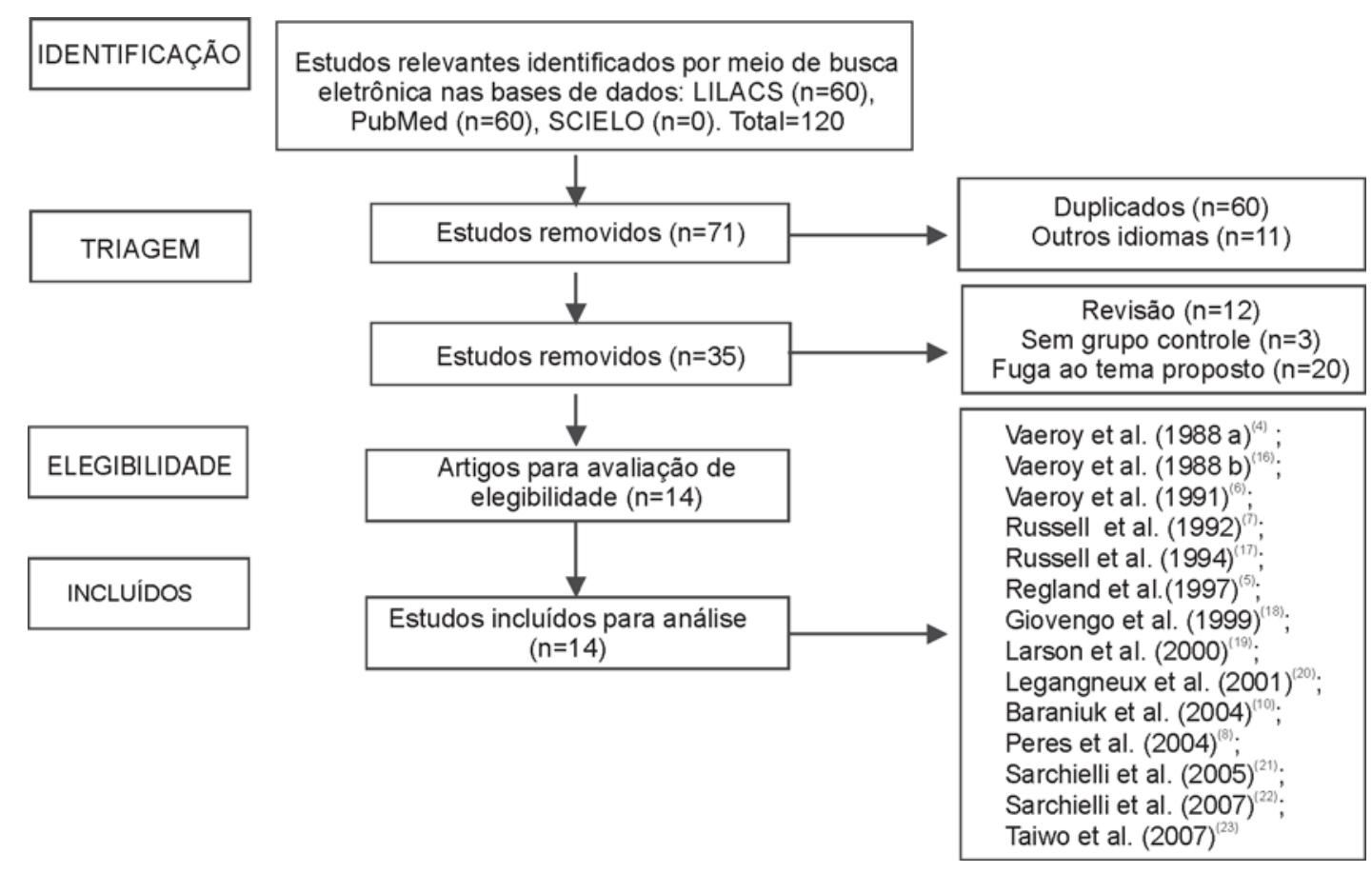

Figura 1. Busca e seleção dos estudos para a revisão 
Todos os estudos selecionados foram anteriores a 2010, de forma que os indivíduos com fibromialgia foram avaliados segundo os critérios de classificação do Colégio Americano de Reumatologia de 1990 (Tabela 1).

Tabela 1. Características dos estudos incluídos

\begin{tabular}{|c|c|c|c|c|}
\hline Autores (Ano) & População & $\begin{array}{l}\text { Substância } \\
\text { analisada }\end{array}$ & $\begin{array}{c}\text { Resultados } \\
\text { FM/Controle } \\
\text { (unidade de medida) }\end{array}$ & $p$ \\
\hline Vaeroy H, Helle R, Forre O, Kass E, et al. $(1988 \mathrm{a})^{(4)}$ & $\begin{array}{l}18 \mathrm{M} \text { com FM } \\
\text { Controles }\end{array}$ & $\beta$-endorfina & $\begin{array}{c}20,7 \pm 0,7 / 20,5 \pm 2 \\
(\mathrm{fmol} / \mathrm{ml})\end{array}$ & $>0,005$ \\
\hline Vaeroy H, Helle R, Forre O, Kass E et al. (1988b) ${ }^{(16)}$ & $\begin{array}{l}30 \text { M com FM } \\
\text { Controles* }\end{array}$ & Substância P & $\begin{array}{l}36,1 \pm 2,7 / 12,7 \pm 0,7 \\
(\mathrm{fmol} / \mathrm{ml})\end{array}$ & $<0,001$ \\
\hline Vaeroy H, Nyberg F, Terenius L $(1991)^{(6)}$ & $\begin{array}{l}34 \mathrm{M} \text { com FM } \\
14 \text { controles } \\
\text { (6 para dinorfina } \\
8 \text { para MEAP) }\end{array}$ & $\begin{array}{l}\text { Dinorfina-A } \\
\text { MEAP }\end{array}$ & $\begin{array}{c}14,3 \pm 0,9 / 9,3 \pm 2 \\
(\mathrm{fmol} / \mathrm{ml}) \\
35,1 \pm 2,4 / 22,4 \pm 0,95 \\
(\mathrm{fmol} / \mathrm{ml})\end{array}$ & $\begin{array}{l}0,03 \\
<0,05\end{array}$ \\
\hline Russell I, Vaeroy H, Javors M, Nyberg F (1992) ${ }^{(7)}$ & $\begin{array}{c}17 \mathrm{M} \text { com FM } \\
12 \text { controles }(11 \mathrm{M})\end{array}$ & $\begin{array}{l}\text { Serotonina } \\
\text { Norepinefrina } \\
\text { Dopamina }\end{array}$ & $\begin{array}{c}18,19 \pm 1,46 / 29,39 \pm 4,97 \\
(\mathrm{ng} / \mathrm{ml}) \\
5,76 \pm 0,72 / 7,77 \pm 0,77 \\
(\mathrm{ng} / \mathrm{ml}) \\
26,09 \pm 1,9 / 41,89 \pm 4,39 \\
(\mathrm{ng} / \mathrm{ml})\end{array}$ & $\begin{array}{l}0,057 \\
0,028 \\
0,005\end{array}$ \\
\hline Russell I, Orr M, Littman B, Vipraio G, et al.(1994)(17) & $\begin{array}{l}32 \text { indivíduos com } \\
\text { FM }(26 \mathrm{M}) \\
30 \text { controles }(17 \mathrm{M})\end{array}$ & Substância P & $\begin{array}{c}42,8 \pm 14,9 / 16,3 \pm 6 \\
(\mathrm{fmol} / \mathrm{ml})\end{array}$ & $<0,001$ \\
\hline $\begin{array}{l}\text { Regland B, Andersson M, Abrahamsson L, } \\
\text { Bagby J, et al. (1997) }\end{array}$ & $\begin{array}{c}11 \mathrm{M} \text { com FM e SFC } \\
18 \text { controles }\end{array}$ & Homocisteína & $\begin{array}{c}0,61 \pm 0,28 / 0,19 \pm 0,04 \\
(\mu \mathrm{mol} / \mathrm{l})\end{array}$ & $<0,0001$ \\
\hline Giovengo S, Russell I, Larson A (1999) ${ }^{(18)}$ & $\begin{array}{c}34 \text { individuos } \\
\text { com FM }(30 \mathrm{M}) \\
35 \text { controles }(18 \mathrm{M})\end{array}$ & $\begin{array}{c}\text { NGF } \\
\text { Substância P }\end{array}$ & $\begin{array}{c}41,8 \pm 12,7 / 9,1 \pm 4,1 \\
\mathrm{pg} / \mathrm{ml} \\
37,4 \pm 2,9 / 17,4 \pm 1,1 \\
\mathrm{pg} / \mathrm{ml}\end{array}$ & $\begin{array}{l}<0,05 \\
<0,05\end{array}$ \\
\hline Larson A, Giovengo S, Russell I, Michalek J (2000) (19) & $\begin{array}{l}16 \text { individuos com } \\
\mathrm{FM}(13 \mathrm{M}) \\
18 \text { controles }(13 \mathrm{M})\end{array}$ & $\begin{array}{l}\text { Arginina } \\
\text { Asparagina } \\
\text { Aspartato } \\
\text { Citrulina } \\
\text { Glutamina } \\
\text { Glutamato } \\
\text { Glicina } \\
\text { Taurina }\end{array}$ & $\begin{array}{c}1,06 \pm 0,09 / 1 \pm 0,07 \\
0,93 \pm 0,07 / 0,93 \pm 0,09 \\
3,55 \pm 0,1 / 3,71 \pm 0,13 \\
1,18 \pm 0,12 / 1,48 \pm 0,25 \\
76,54 \pm 5,62 / 72,16 \pm 5,63 \\
0,29 \pm 0,03 / 0,27 \pm 0,03 \\
0,89 \pm 0,08 / 0,77 \pm 0,09 \\
6,21 \pm 0,57 / 5,9 \pm 0,54 \\
(\mathrm{mg} / \mathrm{l})\end{array}$ & $\begin{array}{l}>0,05 \\
>0,05 \\
>0,05 \\
>0,05 \\
>0,05 \\
>0,05 \\
>0,05 \\
>0,05\end{array}$ \\
\hline $\begin{array}{l}\text { Legangneux E, Mora J, Spreux-Varoquaux O, } \\
\text { Thorin I, et al. }(2001)^{(20)}\end{array}$ & $\begin{array}{l}30 \text { individuos com } \\
\mathrm{FM}(24 \mathrm{M}) \\
69 \text { controles }(31 \mathrm{M})\end{array}$ & $\begin{array}{l}\text { Serotonina } \\
\text { Norepinefrina } \\
\text { Dopamina }\end{array}$ & $\begin{array}{c}21,54 \pm 1,58 / 25,46 \pm 1,27 \\
8,33 \pm 0,33 / 9,89 \pm 0,31 \\
35,01 \pm 2,87 / 36,37 \pm 1,66 \\
(\mathrm{ng} / \mathrm{ml})\end{array}$ & $\begin{array}{l}0,618 \\
0,003 \\
0,172\end{array}$ \\
\hline Baraniuk J, Whalen G, Cunninham J, Clauw (2004) ${ }^{(10)}$ & $\begin{array}{l}14 \text { individuos com } \\
\text { FM }(13 \mathrm{M}) \\
6 \text { controles }(4 \mathrm{M})\end{array}$ & $\begin{array}{c}\text { MEAP } \\
\text { Nociceptina }\end{array}$ & $\begin{array}{c}101,7 / 26,3 \\
4,27 / 5,65 \\
(\mathrm{pg} / \mathrm{ml})\end{array}$ & $\begin{array}{l}<0,01 \\
>0,05\end{array}$ \\
\hline Peres M, Zukerman E, Soares C, Alonso E, et al.(2004) ${ }^{(8)}$ & $\begin{array}{l}12 \text { individuos com } \\
\text { migrânea e FM ( } 9 \mathrm{M}) \\
\text { Controles sem FM }\end{array}$ & Glutamato & $\begin{array}{c}0,34 \pm 0,27 / 0,19 \pm 0,06 \\
(\mu \mathrm{mol} / \mathrm{l})\end{array}$ & $<0,04$ \\
\hline \multirow[t]{2}{*}{ Sarchielli P, Alberti A, Candelieri A, Floridi A, et al. (2005) ${ }^{(21)}$} & $\begin{array}{l}20 \text { individuos com } \\
\text { FM }(15 \mathrm{M})\end{array}$ & GDNF & $31,1 \pm 2,2 / 51,4 \pm 6,5$ & $<0,0008$ \\
\hline & 20 controles & Somatostatina & $\begin{array}{c}50,1 \pm 4,1 / 69,7 \pm 3,2 \\
(\mathrm{pg} / \mathrm{ml})\end{array}$ & $<0,001$ \\
\hline \multirow[t]{2}{*}{ Sarchielli P, Mancini M, Floridi A, Coppola F, et al. (2007) ${ }^{(22)}$} & $\begin{array}{c}20 \text { individuos com } \\
\mathrm{FM}(17 \mathrm{M}) \\
20 \text { controles }(15 \mathrm{M})\end{array}$ & $\begin{array}{l}\text { NGF } \\
\text { BDNF }\end{array}$ & $\begin{array}{l}47,2 \pm 5,3 / 13,7 \pm 2,7 \\
40,4 \pm 4,6 / 11,3 \pm 3,4\end{array}$ & $\begin{array}{l}<0,001 \\
<0,001\end{array}$ \\
\hline & & Glutamato & $\begin{array}{c}2,36 \pm 0,3 / 1,37 \pm 0,3 \\
(\mu \mathrm{mol} / /)\end{array}$ & $<0,003$ \\
\hline Taiwo O, Russell I, Mignot E, Lin L, et al. (2007)(23) & $\begin{array}{l}25 \text { individuos com } \\
\text { FM }(24 \mathrm{M}) \\
25 \text { controles }(22 \mathrm{M})\end{array}$ & Hipocretina-1 & $\begin{array}{c}300,2 \pm 32,2 / 286,5 \pm 34,3 \\
(\mathrm{pg} / \mathrm{ml})\end{array}$ & $>0,05$ \\
\hline
\end{tabular}

M (mulher); FM (fibromialgia); MEAP (Met-encefalina-Arg ${ }^{6}-\mathrm{Phe}^{7}$ ); GDNF (fator neurotrófico derivado da glia); NGF (fator de crescimento neuronal); BDNF (fator neurotrófico derivado do cérebro). *Estudo de Berrettini et al. 1985 
Agrupando-se todos os estudos foi possível observar que foram analisados 22 neurotransmissores ou neuromoduladores. $\bigcirc$ grupo fibromialgia foi composto por 158 indivíduos, dos quais $80 \%$ eram mulheres com idade superior a 18 anos. Os grupos controles foram compostos por indivíduos saudáveis.

\section{DISCUSSÃO}

Nesta revisão, foi possível observar que muitos neurotransmissores e neuropeptídios estão relacionados com quadros de dor crônica, como a fibromialgia e também foi encontrado que, de forma semelhante à literatura, há uma predileção da doença pelo gênero feminino.

O quadro clínico de dor encontrado na fibromialgia sugere que possa existir um aumento de atividade ou hipersensibilidade nas vias nociceptivas ou ainda alterações que levam a atividade inadequada dos mecanismos de analgesia. (6) No sistema endógeno de analgesia há participação pelos sistemas neuronais, envolvendo endorfinas, encefalinas e dinorfinas. ${ }^{(24)}$ Estudo de Vaeroy e colaboradores (1988)(4) iniciou as pesquisas com peptídeos opioides em indivíduos com fibromialgia, onde foi possível observar que as concentrações de $\beta$-endorfinas no LCR de indivíduos com fibromialgia eram equivalentes às concentrações encontradas em indivíduos saudáveis, 0 que os levou a concluir que, além da $\beta$-endorfina, outros peptídeos opioides, como a encefalina e a dinorfina, também poderiam estar envolvidas nos mecanismos de analgesia da fibromialgia. Outro estudo(b) desse mesmo grupo investigou como se comportavam as concentrações da Met-encefalina-Arg ${ }^{6}-\mathrm{Phe}^{7}$ (MEAP) e da dinorfina A no LCR de mulheres com fibromialgia. Ambas as substâncias estavam elevadas sugerindo que existe uma hipersecreção de opióides, que dessensibilizariam os receptores, levando a uma diminuição da capacidade de modulação da dor. No estudo de Baraniuk e colegas, ${ }^{(10)}$ encontrou-se concentração de MEAP três vezes maior em indivíduos com fibromialgia quando comparados com indivíduos normais. Opostamente, o estudo de Liu e colegas ${ }^{(25)}$ mostrou baixos níveis de MEAP em indivíduos com fibromialgia. Talvez essa diferença tenha acontecido pela diferença de método na análise da substância estudada.

A redução da capacidade de modulação da dor, decorrente do aumento dos níveis de dinorfina e encefalina, leva a inibição pré-sináptica nos neurônios que secretam substância P no corno dorsal da medula, acarretando em um aumento da liberação de substância $P^{(24)}$

A substância P é um peptídeo com 11 aminoácidos, que atua como neurotransmissor e neuromodulador, e tem a função de conduzir estímulos álgicos para $\circ$ SNC. ${ }^{17,26)}$ As funções fisiológicas da substância P sofrem influencia da serotonina. ${ }^{(27)}$ Estudo de Vaeroy e colaboradores, ${ }^{(16)}$ com mulheres com fibromialgia, mostrou que os níveis de substância P eram maiores nestas, quando comparadas a um grupo controle saudável. Os achados de Russell e colegas ${ }^{(17)}$ confirmaram os achados anteriores. Não existe uma explicação para os níveis aumentados de substância $\mathrm{P}$ em pacientes com fibromialgia, mas considerando sua estreita relação com a nocicepção, pode-se dizer que qualquer defeito em sua produção, atividade funcional ou degradação podem levar a percepção anormal da dor. ${ }^{(17)}$ Dessa forma, supõe-se que a substância $P$ seja um dos responsáveis pela hiperalgesia na fibromialgia. ${ }^{(4)}$

A síntese da substância $P$ nas fibras $C$ aferentes primárias é regulada pelo NGF.(19) Estudos experimentais em modelos animais vêm demonstrando o papel nas neurotrofinas nos mecanismos fisiopatológicos da hiperalgesia ${ }^{(28)}$ e da dor crônica. ${ }^{(29)}$ Estudo de Giovengo e colegas $^{(18)}$ mostrou que os níveis de NGF em pacientes com fibromialgia eram altos, o que levou a se pensar no NGF como um dos responsáveis pela sintomatologia dolorosa da fibromialgia. Resultados semelhantes foram encontrados por Sarchielli e colaboradores, ${ }^{(22)}$ que, além de avaliarem o NGF, avaliaram também o BDNF e glutamato, e encontraram níveis elevados de ambos no LCR de pacientes com fibromialgia. Em outro estudo realizado por Sarchielli e colegas, ${ }^{(21)}$ foram analisadas as substâncias GDNF e somatostatina e as mesmas encontravam-se em níveis menores no LCR de indivíduos com fibromialgia.

Os fatores neurotróficos parecem influenciar em alterações anatômicas, neuroquímicas e funcionais de neurônios sensoriais e trigeminais na fibromialgia, de modo que se tem atribuído ao NGF o papel da sensibilização periférica dos nociceptores e ao BDNF a modulação central da dor. ${ }^{(22)}$ Em humanos adultos, o NGF também tem demonstrado sua função na hiperalgesia. ${ }^{(30)}$ O aumento dos níveis de NGF e BDNF leva a uma potenciação da transmissão glutamatérgica, ${ }^{(31)}$ e esse mecanismo contribuiria para a sensibilização central sustentada em estados de dor crônica. ${ }^{(32)}$ Outro fator neurotrófico envolvido na dor é o GDNF, que exerce influência sob a expressão da somatostatina, um neuropeptideo com função antinociceptiva. ${ }^{(33)}$ 
Os aminoácidos excitatórios exercem um papel fundamental na transmissão da dor, sendo o glutamato é o principal deles. Estudos de Peres e colegas ${ }^{(8)}$ mostraram que os níveis de glutamato em pacientes com fibromialgia eram elevados. Os mesmos achados foram encontrados por Sarchielli e colegas. ${ }^{(22)}$ Por outro lado, estudo de Larson e colegas ${ }^{(19)}$ não encontrou alterações nos níveis dos aminoácidos avaliados nos pacientes com fibromialgia. Talvez a pequena amostra tenha interferido nos resultados. Os tender points, encontrados na fibromialgia, podem ser considerados alodinia de pressão, provavelmente decorrente da sensibilização central, que pode ser resultado de uma atividade exacerbada do glutamato. ${ }^{(8)}$

Os estudos de Russell e colaboradores ${ }^{(7)}$ e de Legangneux e colegas ${ }^{(20)}$ mostraram que indivíduos com fibromialgia apresentam níveis baixos de serotonina, porém sem diferença significativa em relação ao grupo controle noradrenalina e dopamina no LCR. $O$ estudo de Legangneux e colaboradores ${ }^{(20)}$ ainda demonstrou que os níveis de serotonina plaquetária estão elevados, sugerindo que uma deficiência funcional da serotonina pode estar envolvida no mecanismo fisiopatológico da fibromialgia. A ocorrência de deficiência na noradrenalina e na dopamina, simultaneamente à serotonina, sugere que a deficiência da serotonina é apenas parte de um processo ainda maior. ${ }^{(7)} A$ redução nos níveis centrais de aminas biogênicas pode aumentar a sensibilidade a estímulos dolorosos, uma característica importante da fibromialgia. A serotonina é um mediador da percepção da dor, de alterações psiquiátricas, da função intestinal e também do sono profundo, podendo gerar manifestações típicas da fibromialgia. ${ }^{(20)}$

Estudos que relacionam a hipocretina à fibromialgia ainda são escassos. Apenas um artigo foi encontrado e nesse, os níveis da substância no LCR não foram diferentes dos níveis encontrados no grupo controle. A hipocretina é um neuropeptídeo que provoca alterações no sono, uma característica marcante na fibromialgia. A administração central da hipocretina também apresenta efeito analgésico. ${ }^{(23)} \mathrm{Em}$ estudos experimentais com animais, a privação aguda do sono aumentou os níveis de hipocretina no LCR. ${ }^{(34,35)} \mathrm{Na}$ fibromialgia, observamos que muitos pacientes apresenta sonolência diurna, mas esse fator não apresentou associação com alterações nos níveis de hipocretina. ${ }^{(23)}$

Assim como a hipocretina, a homocisteína também é pouco estudada. $\bigcirc$ único artigo encontrado sobre o tema mostrou que indivíduos com fibromialgia apre- sentavam níveis de hosmocisteina no LCR maiores que o grupo controle. $O$ estudo concluiu que esses níveis elevados não são específicos da fibromialgia, sendo uma característica comum a várias doenças neuropsiquiátricas.

Resumidamente, foi possível observar os seguintes comportamentos das substâncias com funções neurotransmissoras ou neuromoduladoras no LCR:

1) Níveis aumentados: substância $P$, dinorfina $A$, MEAP, Homocisteína, NGF, glutamato e BDNF;

2) Níveis diminuídos: noradrenalina, dopamina, serotonina, GDNF e somatostatina;

3) Níveis inalterados: hipocretina, $\beta$-endorfina e, aminoácidos excitatórios.

\section{CONCLUSÃO}

A presente revisão mostrou o comportamento de algumas substâncias encontradas no SNC ou a ele ligadas em pacientes com fibromialgia. Apesar de ser uma doença de etiologia incerta, foi possível observar que muitas das substâncias estudadas apresentam relação com a fibromialgia, o que corrobora com algumas pesquisas que definem a fibromialgia como uma doença do SNC, seja por apresentar alterações no sistema neuroendócrino e sistema nervoso autônomo, ou por apresentar alterações nas concentrações de neurotransmissores e neuropeptídios no LCR.

\section{REFERÊNCIAS}

1. Wolfe F, Anederson J, Russell IJ, et al. The prevalence and characteristics of fibromyalgia in general population. Arthritis Rheum. 1995;38(1):19-28.

2. Clauwn DJ. Fibromyalgia: an overview. Am J Med. 2009; 122(12) Suppl 1,S3-S13.

3. Valença MM, Medeiros FL, Martins HA, et al. Neuroendocrine dysfunction in fibromyalgia and migraine. Curr Pain Headache Rep. 2009;13:358-64.

4. Vaeroy H, Helle R, Forre $O$, et al. Cerebrospinal fluid levels of ?endorphin in patients with fibromyalgia (fibrositis syndrome). J Rheumatol. 1988;5:12. (a)

5. Regland B, Andersson M, Bagby J, et al. Increased concentratios of homocysteine in the cerebrospinal fluid in patients with fibromyalgia and chronic fatigue syndrome. Scand J Rheumatol. 1997;26:301-7.

6. Vaeroy H, Nyberg F, Terenius L. No evidence for endorphin deficiency in fibromyalgia following investigation of cerebrospinal fluid (CSF) dynorphin A and Met-enkephalin-Arg6-Phe7. Pain. $1991 ; 46: 139-43$

7. Russell IJ, Vaeroy H, Javors M, Nyberg F. Cerebrospinal fluid biogenic amine metabolites in fibromyalgia/fibrositis syndrome and rheumatoid arthritis. Arthritis Rheum. 1992;35(5):550-6. 
8. Peres MF, Zukerman E, Senne Soares CA, et al. Cerebrospinal fluid glutamate levels in chronic migraine. Cephalalgia. 2004; 24(9):735-9.

9. Vaerøy H, Sakurada T, Førre O, et al. Modulation of pain in fibromyalgia (fibrositis syndrome): cerebrospinal fluid (CSF) investigation of pain related neuropeptides with special reference to calcitonin gene related peptide (CGRP). J Rheumatol Suppl. 1989;19:94-7.

10. Baraniuk JN, Whalen G, Cunningham J, Clauw DJ. Cerebrospinal fluid levels of opioid peptides in fibromyalgia and chronic low back pain. BMC Musculoskelet Disord. 2004;5(48).

11. Adler GK, Geenen R. Hypothalamic-pituitary-adrenal and autonomic nervous system functioning in fibromyalgia. Rheum Dis Clin N Am. 2005; 31 (1):187-202.

12. Robinson ME, Craggs JG, Prince DD et al. Gray matter volumes of pain-related brain areas are decreased in fibromyalgia syndrome. J Pain. $2011 ; 12(4): 436-43$.

13. Bradley LA. Pathophysiology of fibromyalgia. Am J Med. 2009; 22(12) Suppl 1:S22-S30.

14. Wolfe F, Smythe HA, Yunus MB et al. The American College of Rheumatology 1990 criteria for the Classification of fibromyalgia. Report of the multicenter criteria committee. Arthritis Rheum. 1990;3:160-72.

15. Wolfe F, Clauwn DJ, Fitzcharles M, et al. Fibromyalgia criteria and severity scales for clinical and epidemiological studies: a modification of the ACR preliminary diagnostic criteria for fibromyalgia. J Rheumatol. 2010;38(6):1113-22.

16. Vaerøy $H$, Helle R, Førre $O$, et al. Elevated CSF levels of substance $P$ and high incidence of Raynaud phenomenon in patients with fibromyalgia: new features for diagnosis. Pain. 1988;32(1):21-6. (b)

17. Russell IJ, Orr MD, Littman B, et al. Elevated cerebrospinal fluid levels of substance $P$ in patients with the fibromyalgia syndrome. Arthritis \& Rheumatism. 1994;37(1 1):1593-1601.

18. Giovengo SL, Russell IJ, Larson AA. Increased concentrations of nerve growth factor in cerebrospinal fluid of patients with fibromyalgia. J Rheumatol. 1999; 26(7).

19. Larson AA, Giovengo SL, Russell IJ, Michalek JE. Changes in the concentration of amino acids in the cerebrospinal fluid that correlate with pain in patients with fibromyalgia: implications for nitric oxide pathways. Pain. 2000; 87:201-11.

20. Legangnaux E, Mora JJ, Spreux-Varoquaux O, et al. Cerebrospinal fluid biogenic amine metabolites, plasma-rich platelet serotonina and $[3 \mathrm{H}]$ imipramine reuptake in the primary fibromyalgia syndrome. Rheumatology. 2001 ;40:290-6.

21. Sarchielli P, Alberti A, Candelieri A, et al. Glial cell-derived neurotrophic factor and somatostatin levels in cerebrospinal fluid of patients affected by chronic migraine. Cephalalgia. 2005; 26:409-15.

22. Sarchielli $P$, Mancini ML, Floridi A, et al. Increased levels of neurotrophins are not specific for chronic migraine: evidence from primary fibromyalgia syndrome. J Pain. $2007 ; 8(9): 737-45$.

23. Taiwo OB, Russell IJ, Mignot E, et al. Normal cerebrospinal fluid levels of hypocretin-1 (orexin A) in patients with fibromyalgia syndrome. Sleep Medicine. 2007;8:260-65.
24. Ramanathan S, Panksepp J, Johnson B. Is fibromyalgia an endocrine/endorphin deficit disorder? Is low dose naltrexone a new treatment option? Psychosomatics. 2012; 53:591-4.

25. Liu Z Welin M, Bragee B, Nyberg F. A high-recovery extraction procedure for quantitative analysis of substance $P$ and peptides in human cerebrospinal fluid. Peptides. 2000; 21 (6):853-60.

26. Gur A, Oktayoglu P. Central nervous system abnormalities in fibromyalgia and chronic fatigue syndrome: new concepts in treatment. Curr Pharm Des 2008, 14(13):1274-94.

27. Murphy RM, Zemlan FP. Differential effects of substance P on serotonin-modulated spinal nociceptive reflexes. Psychopharmacology (Berlin). 1987; 93:118-21.

28. Gould HJ, Gould TN, England JD, et al. A possible role for nerve growth factor in the augmentation of sodium channels in models of chronic pain. Brain Res. 2000; 854(1-2): 19-29.

29. Melemediian OK, Tillu DV, Asiedu MN, et al. BDNF regulates atypical PKC at spinal synapses to initiate and maintain a centralized chronic pain state. Mol Pain. 2013; 20(9):12.

30. Lewin GR, Mendell LM. Nerve growth factor and nociception. Trends Neurosci. 1993; 13:2136:48.

31. Sarchielli P, Alberti A, Gallai B, et al. Brain-derived neurotrophic factorin cerebrospinal fluid of patients with chronic daily headache: relationship with nerve growth factor and glutamate levels. J Headache Pain. 2002; 4:1 15-27.

32. Nygraha B, Engeli S, Gutenbrunner C. Brain-derived neurotrophic factor and exercise in fibromyalgia syndrome patients: a mini review. Rheumatol Int. 2012; 32(9): 2593-9.

33. Malcangio M. GDNF and somatostatin in sensory neurons. Curr Opin Pharmacol. 2003; 3:41-5

34. Yoshida Y, Fujiki N, Nakajima T, et al. Fluctuation of extracellular hypocretin-1 (orexin A) levels in the rat in relation to the lightdark cycle and sleep-wake activities. Eur J Neurosci. 2001; 14:1075-81.

35. Zeitzer JM, Buckmaster CL, Parker KJ, et al. Circadian and homeostatic regulation of hypocretin in a primate model: implications for the consolidation of wakefulness. J Neurosci. 2003; 23: 3555-60.

\section{Correspondência \\ Lovana Cassiano da S. Lima \\ Rua 9 de Janeiro, 92A, Centro 55660-000 - Bezerros, PE lovana_cs@hotmail.com}

Recebido: 3 julho 2014

Aceito: 2 novembro 2014 